\title{
TEM and HAADF STEM Imaging of Dislocation Loops in Irradiated GaAs
}

\author{
J.H. Neethling ${ }^{a, *}$, A. Janse Van Vuuren ${ }^{a}$, E.J. Olivier ${ }^{a}$ And P.A. Van Aken ${ }^{b}$ \\ ${ }^{a}$ Centre for HRTEM, Nelson Mandela University, Port Elizabeth, South Africa \\ ${ }^{b}$ Stuttgart Center for Electron Microscopy, Max Planck Institute for Solid State Research, Stuttgart, Germany
}

This paper compares earlier transmission electron microscopy results of dislocation loops generated in $n$-type GaAs by $1 \mathrm{MeV}$ electron and fast neutron irradiation with recent Cs-corrected high angle annular dark field scanning transmission electron microscopy imaging of $\{110\}$ and $\{111\}$ dislocation loops in neutron irradiated and annealed $\left(600^{\circ} \mathrm{C}\right.$ for $\left.20 \mathrm{~min}\right) n$-type GaAs. High angle annular dark field scanning transmission electron microscopy revealed that the $\{110\}$ loop plane consists of two layers of Ga and As atoms, which is consistent with the model for a $\{110\}$ pure-edge interstitial dislocation loop in GaAs. The loop plane of the $\{111\}$ interstitial loop consists of one layer of $\mathrm{Ga}$ and As atoms while the stacking sequence across the loop is consistent with that of an extrinsic stacking fault. The projected positions of the Ga and As atom columns across the extrinsic stacking fault plane of the $\{111\}$ loop indicate polarity continuation across the loop and the preservation of charge neutrality. The annealing stage at $500{ }^{\circ} \mathrm{C}$ found to be the minimum temperature for small dislocation loop formation visible in transmission electron microscopy, agrees with the final annealing stage of $400-600^{\circ} \mathrm{C}$ for the electrical conductivity of electron irradiated $n$-type GaAs. It is proposed that $\{110\}$ loops in proton bombarded GaAs are the same type of interstitial loops found in neutron irradiated GaAs while hydrogen platelets form on $\{111\}$ planes in GaAs exposed to a hydrogen plasma at $180^{\circ} \mathrm{C}$ or in high-dose proton bombarded GaAs.

DOI: 10.12693/APhysPolA.136.245

PACS/topics: 61.72.Ff, 61.72.Nn, 61.72.uj

\section{Introduction}

Gallium arsenide (GaAs) is a very promising semiconductor material for space solar cells — it has a higher conversion efficiency and better radiation resistance than silicon $(\mathrm{Si})[1,2]$. Solar cells used in space are exposed to severe radiation and the lattice defects induced by highenergy electron and proton irradiations cause a decrease in the power output of the solar cells [1]. The study of radiation damage in GaAs therefore remains relevant since a thorough understanding of the mobility and agglomeration behavior of irradiation induced point defects in GaAs is important for its widespread use in space missions where higher conversion efficiencies and radiation resistance are required. Although the first author of this paper has investigated radiation damage and the formation and growth of dislocation loops in $n$-type GaAs since the 1980s [3-5], we are not aware of any transmission electron microscopy (TEM) study of neutron irradiated GaAs published to date. The earlier TEM studies of radiation damage in proton bombarded GaAs [3-5] were also done long before probe-Cs-corrected high angle annular dark field (HAADF) scanning transmission electron microscopy (STEM) imaging became available and hence it was challenging to distinguish between an interstitial dislocation loop and a hydrogen platelet without being able to resolve the atomic structure of the loop or platelet directly.

*corresponding author; e-mail: Jan.Neethling@mandela.ac.za
In earlier papers, the first author (J.H.N.) and co-workers concluded that the loop-like defects on $\{110\}$ planes present in $300 \mathrm{keV}$ proton bombarded $\left(10^{15}\right.$ to $\left.10^{16} \mathrm{H}^{+} \mathrm{cm}^{-2}\right) n$-type Si-doped GaAs annealed in the range $500^{\circ} \mathrm{C}$ to $900^{\circ} \mathrm{C}$ were hydrogen filled vacancy loops (called hydrogen platelets) [3-5] which, due to the gas pressure inside the loops, would appear to be of interstitial nature [3]. This conclusion was supported by still earlier reports of vacancy loops on $\{110\}$ planes observed in Si- and Te-doped GaAs annealed at high temperatures $[6-8]$ and the fact that for low dose electron irradiated $n$-type GaAs, pointdefect annealing already occurs in the temperature range 200 to $300^{\circ} \mathrm{C}$, which is evidence for point defect mobility in this temperature range [9]. The new data presented in this paper allows for a reinterpretation of the older defect models in proton bombarded $n$-type GaAs, i.e. the $\{110\}$ loops in proton bombarded and annealed GaAs are now considered to be interstitial dislocation loops while hydrogen platelets form on $\{111\}$ planes in highdose proton bombarded GaAs $\left(5 \times 10^{16}-10^{17}\right)$ annealed in the range $180-230^{\circ} \mathrm{C}$.

The development of probe-Cs-corrected STEMs with the HAADF imaging mode has provided a powerful technique for determining the structural and chemical information at atomic resolution. This technique has recently been used by Olivier and co-workers to solve the longstanding problem of the atomic structure and local chemistry of $\{001\}$ platelet defects in type Ia diamond [10]. The current paper compares earlier TEM results of dislocation loops generated in $n$-type GaAs by $1 \mathrm{MeV}$ electron and fast neutron irradiation with recent Cs-corrected 
HAADF STEM imaging of $\{110\}$ and $\{111\}$ dislocation loops in neutron irradiated and annealed $n$-type GaAs. The annealing stage where radiation induced interstitial atoms coalesce to form dislocation loops visible in TEM, is correlated qualitatively with the final annealing stage of the electrical conductivity of electron irradiated $n$-type GaAs [9].

\section{Experimental details}

Electron irradiation of (001) $n$-type Si-doped $\left(1 \times 10^{18}\right.$ carriers $\left./ \mathrm{cm}^{3}\right)$ GaAs samples was done in an AEI EM7 high voltage electron microscope (HVEM) at an accelerating voltage of $1 \mathrm{MV}$ (Max Planck Institute, Stuttgart). The HVEM was also used for in situ observation of the evolution of radiation damage with annealing temperature in the range -223 to $500^{\circ} \mathrm{C}$. The neutron irradiation of (001) n-type GaAs wafers, to a total neutron fluence of $2.8 \times 10^{20}$ neutrons $/ \mathrm{cm}^{2}$, was carried out at the nuclear energy corporation of South Africa (NECSA). The neutron irradiated samples were annealed in a furnace at temperatures of 600 to $900{ }^{\circ} \mathrm{C}$, for $20 \mathrm{~min}$ in flowing argon. Final TEM foil thinning was done by using argon ion milling in a Gatan PIPS system. For initial conventional TEM investigations, Philips EM 420 and CM20 TEMs were used. HRSTEM specimens were prepared by using a Helios Nanolab 650 focused ion beam (FIB) SEM. HAADF STEM imaging was carried out using a double Cs-corrected JEOL ARM 200F. Electron energy loss (EEL) spectrum imaging, used to map the positions of $\mathrm{Ga}$ and As atom columns across a $\{111\}$ dislocation loop, was performed using a probe-Cs-corrected JEOL ARM 200F (at the Max Planck Institute in Stuttgart). The experimental parameters used for STEM spectrum imaging are the following: probe diameter $\approx 0.09-0.1 \mathrm{~nm}$, convergence angle $21 \mathrm{mrad}$, spectrometer entrance aperture acceptance angle $\approx 82 \mathrm{mrad}$, pixel dwell time $0.02 \mathrm{~s}$, and pixel size (step size) $0.04 \mathrm{~nm}$. The spectrometer was operated in Dual EELS mode, with a dispersion of $1 \mathrm{eV} / \mathrm{ch}$ and high spectrum energy window 600-2620 eV energy loss.

\section{Results}

The preliminary results of the in situ electron irradiated and annealed $n$-type GaAs was reported earlier in [11]. A (001) $n$-type Si-doped $\left(1 \times 10^{18}\right.$ carriers $\left./ \mathrm{cm}^{3}\right)$ GaAs TEM foil was irradiated with $1 \mathrm{MeV}$ electrons at a temperature of $-223^{\circ} \mathrm{C}$ and a dose rate of $4 \times 10^{19} \mathrm{e} /\left(\mathrm{cm}^{2} \mathrm{~s}\right)$. The electron beam direction was close to the [001] with $\boldsymbol{g}=220$ and $\boldsymbol{s} \approx 0$. Radiation damage in the form of dark diffuse clusters became visible after $8 \mathrm{~min}$ of irradiation as shown in Fig. 1a. The sample was then allowed to heat up slowly from -223 to $17^{\circ} \mathrm{C}$ while it was observed in situ, but no evidence of dislocation loop formation was found. By using the same dose rate as before, GaAs was irradiated at room temperature and small defect clusters became visible after $10 \mathrm{~min}$. The temperature was then slowly increased and a dramatic and rapid change in the defect cluster structures took place at $500^{\circ} \mathrm{C}$ with the formation of small dislocation loops on $\{110\}$ and $\{111\}$ planes of which a typical example is shown in Fig. 1b. In order to determine the vacancy/interstitial nature of the loops, a (110) GaAs sample was irradiated at $25^{\circ} \mathrm{C}$ using the same conditions as before, and then annealed in a furnace at $800^{\circ} \mathrm{C}$ for 4 min under argon flow. The larger loops on $\{110\}$ planes were analysed using the inside/outside contrast method and found to be of interstitial nature as reported earlier [11].
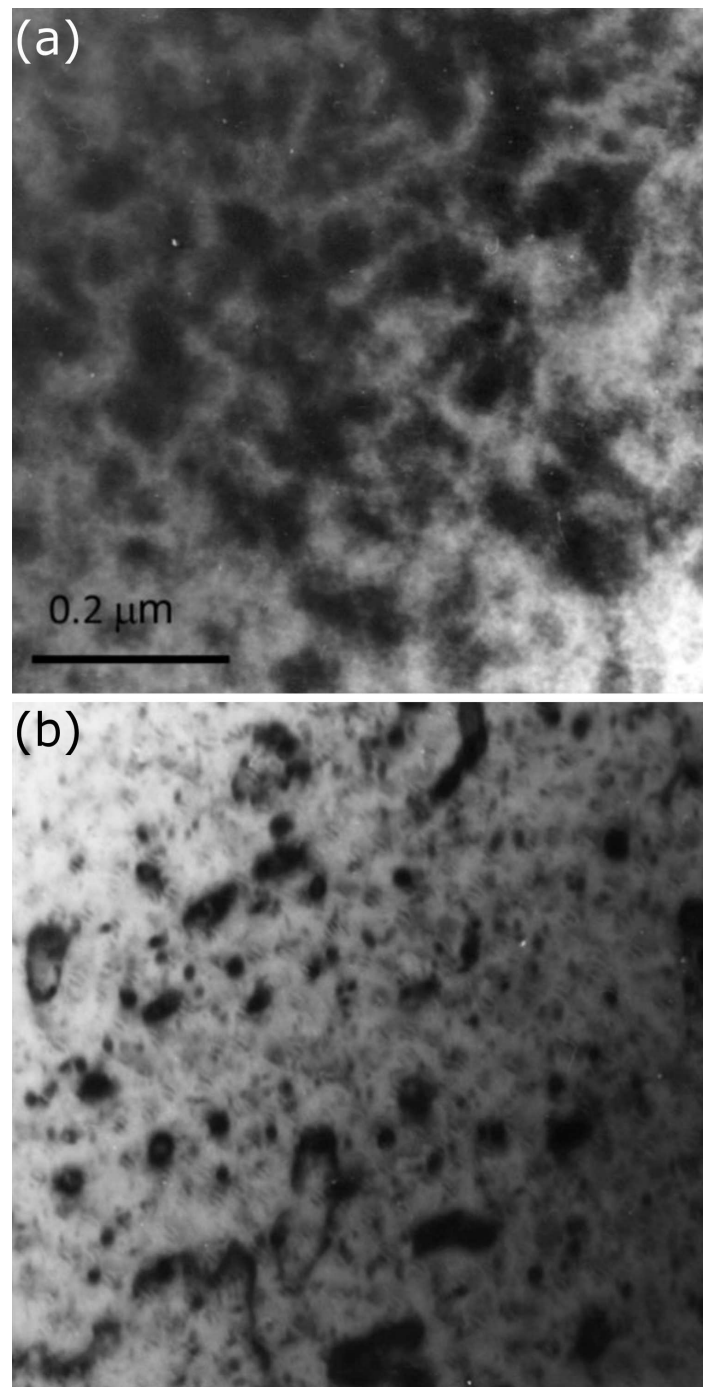

Fig. 1. Bright field HVEM images of $1 \mathrm{MeV}$ electron irradiated GaAs showing the radiation induced defect structure at (a) $-223^{\circ} \mathrm{C}$ and (b) $500{ }^{\circ} \mathrm{C}$. The beam direction is close to $\langle 001\rangle$.

A comparison of the radiation damage in proton and electron irradiated $n$-type GaAs is not trivial since the defects formed in proton irradiated and annealed GaAs consist of interstitial $\{110\}$ loops [3], \{111\} hydrogen platelets and planar void/gas bubble rafts [12]. 
Therefore neutron irradiated GaAs was used to study radiation induced dislocation loops without the additional contributions of hydrogen as found in proton irradiated GaAs. (001) n-type GaAs wafers were irradiated to a total fast-neutron fluence of $2.8 \times 10^{20}$ neutrons $/ \mathrm{cm}^{2}$ and annealed in the range 600 to $900^{\circ} \mathrm{C}$ for $20 \mathrm{~min}$. Bright field TEM investigations of the neutron irradiated and annealed GaAs revealed a high density of dislocation loops as shown in Fig. 2a and b for annealing temperatures of 600 and $800^{\circ} \mathrm{C}$, respectively. Larger loops on $\{110\}$ planes were analysed using the method of inside/outside contrast (see Fig. 3) and found to be of interstitial nature.

Cs-corrected HAADF STEM was used to image small $\{110\}$ and $\{111\}$ dislocation loops in neutron irradiated GaAs annealed at $600^{\circ} \mathrm{C}$. Figure 4 shows a HAADF STEM image of an interstitial loop on a $\{110\}$ plane viewed edge on. A Burgers circuit drawn around the dislocation loop line indicates that the loop consists
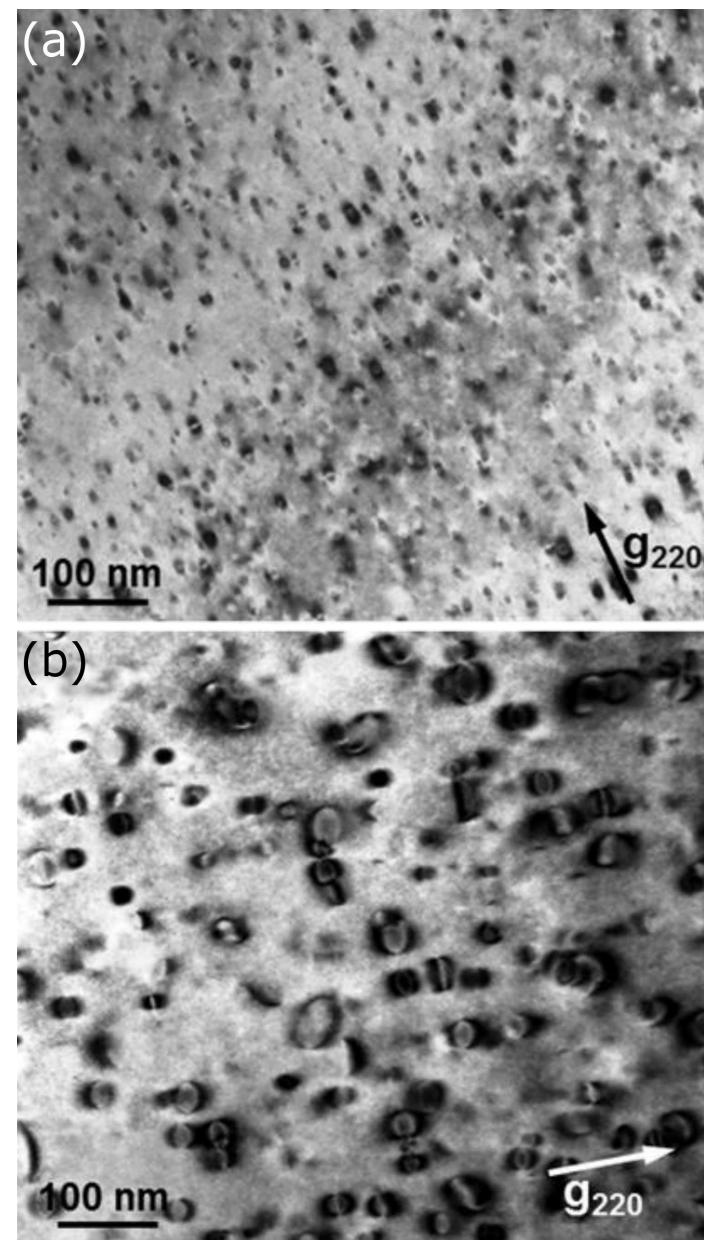

Fig. 2. Bright-field TEM micrographs of neutron irradiated and annealed GaAs at (a) 600 and (b) $80{ }^{\circ} \mathrm{C}$ for $20 \mathrm{~min}$. The loops viewed edge-on on (110) planes perpendicular to the $\boldsymbol{g}=220$ operating reflections, display typical strain contrast images with lines-of-zero contrast perpendicular to the operating $\boldsymbol{g}$-vector. The beam direction is close to [001].
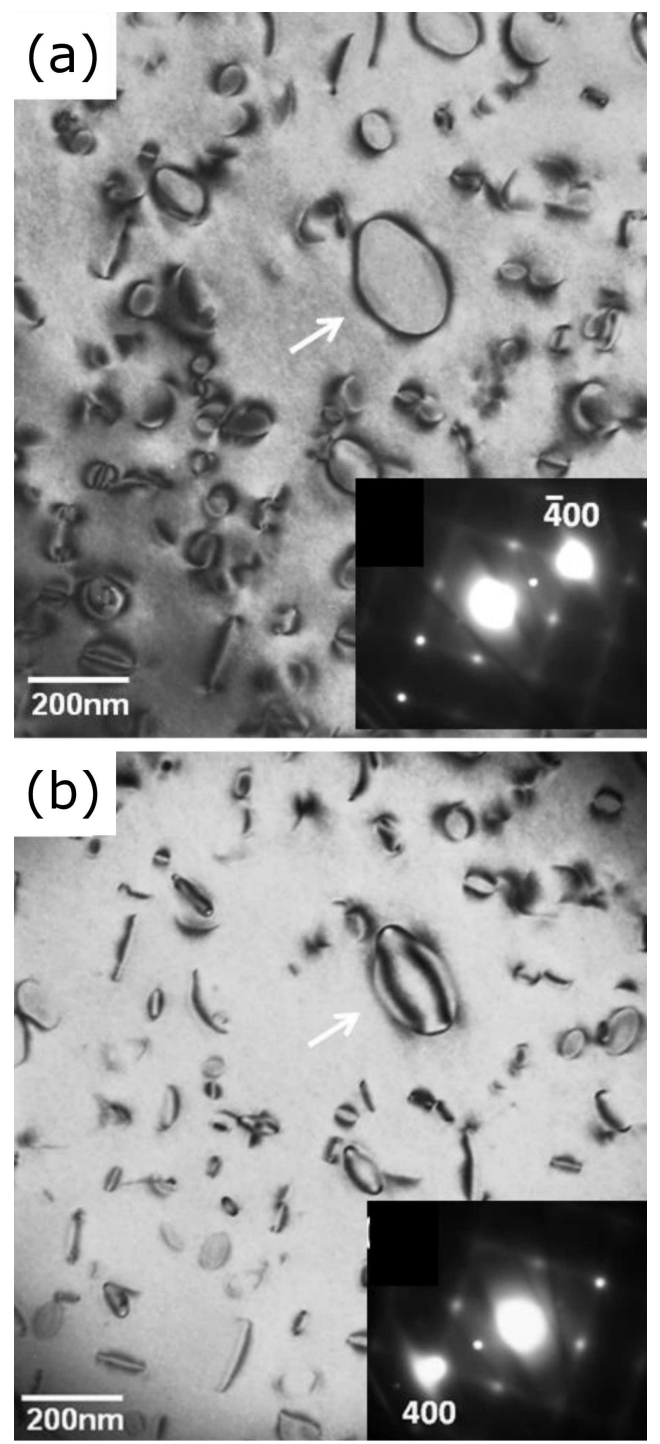

Fig. 3. Determination of the intrinsic/extrinsic nature of dislocation loops in $n$-type GaAs irradiated with neutrons and annealed at $900{ }^{\circ} \mathrm{C}$ for $20 \mathrm{~min}$. (a) The loop indicated by the arrow exhibits outside contrast with inset showing the operating reflection. (b) The loop indicated by the arrow exhibits inside contrast with inset showing the operating vector $\boldsymbol{g}=400$. The electron beam is close to the [001] direction for both (a) and (b) and $s>0$

of a two layers of $\mathrm{Ga}$ and As atoms, which is consistent with the model proposed by Hutchinson and Dobson [13] for a $\{110\}$ pure-edge interstitial dislocation loop in GaAs with a Burgers vector of $b=(a / 2)\langle 110\rangle$. Hence, it is proposed that the $\{110\}$ interstitial dislocation loops found in proton, electron and neutron [3, 11] irradiated $n$-type GaAs annealed at temperatures of $500^{\circ} \mathrm{C}$ and above form by the coalescence of radiation induced $\mathrm{Ga}$ and $\mathrm{As}$ interstitial atoms. In order to preserve charge neutrality, equal numbers of Ga and As interstitials must become mobile and diffuse to sinks, nucleate dislocation loops and grow by the capture of more Ga and As atoms. 


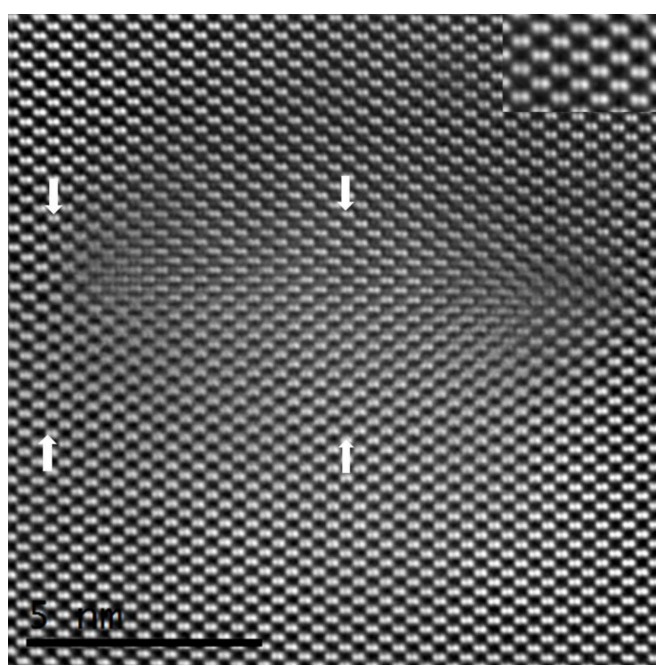

Fig. 4. HAADF STEM image of an interstitial loop on a $\{110\}$ plane in neutron irradiated $n$-type GaAs viewed edge on with the GaAs dumbbells resolved in the $\langle 001\rangle$ direction (see inset). A Burgers circuit drawn around the dislocation loop line indicates that the loop consists of two layers of $\mathrm{Ga}$ and As atoms. The number of planes between the arrows on the left are 23 and between arrows on the right are 25 (through centre of loop). The beam direction is $\langle 110\rangle$.

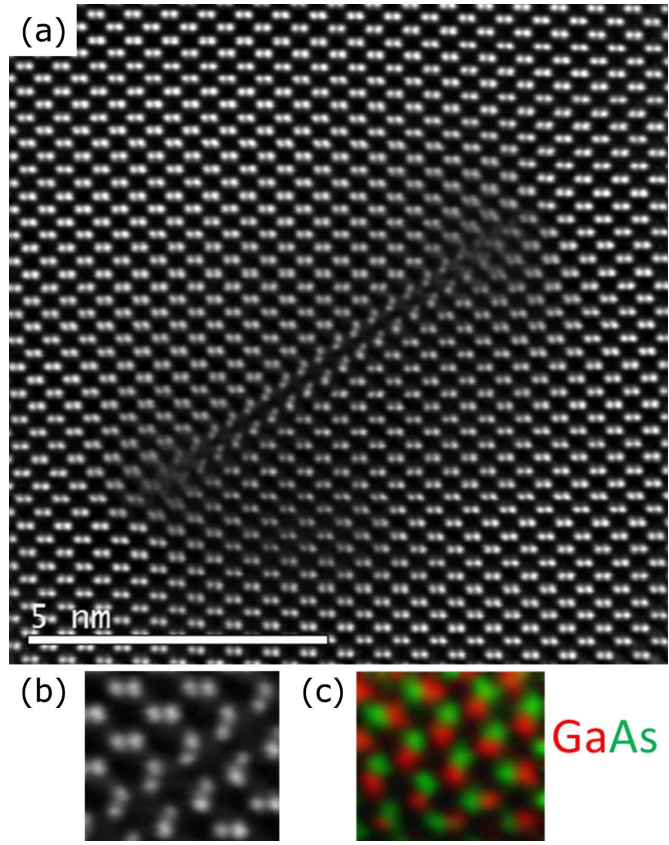

Fig. 5. (a) HAADF STEM image of a small loop on a $\{111\}$ plane in neutron irradiated $n$-type GaAs. The plane of the interstitial loop consists of one layer of $\mathrm{Ga}$ and As atoms while the stacking sequence across the loop is consistent with that of an extrinsic stacking fault. The insertion of a single GaAs rotated layer results in the creation of two modified (rotated) layers. (b) Higher magnification view of $\{111\}$ planes across loop with (c) showing the projected positions of the $\mathrm{Ga}$ and As atom columns across the extrinsic stacking fault plane of the $\{111\}$ loop obtained by using Ga- $L_{2,3}$ and As- $L_{2,3}$ EEL spectrum imaging.
The annealing stage at $500^{\circ} \mathrm{C}$, found to be the minimum temperature for small dislocation loop formation visible in TEM, agrees with the final annealing stage of $400-600^{\circ} \mathrm{C}$ for the electrical conductivity of electron irradiated $n$-type GaAs [9]. In the case of fast neutron irradiated $n$-type GaAs, a final annealing stage for electrical conductivity was found in the range $450-700^{\circ} \mathrm{C}[14]$. Since the annealing stages of the electrical properties of irradiated $n$-type GaAs were found to be dependent on the radiation induced point defect concentrations [9], annealing stages of electrical properties reported for electron and neutron irradiated $n$-type GaAs differed [14, 15$]$. For electron irradiated $n$-type GaAs, it was proposed that the annealing stages of electrical properties comprise firstly of point defect annealing and clustering that takes place at about $200-300^{\circ} \mathrm{C}$, and secondly the annealing of clusters which take place in the $400-600^{\circ} \mathrm{C}$ temperature range [9]. Although the authors of the current paper associate the formation of dislocation loops observed by TEM at $500{ }^{\circ} \mathrm{C}$ and above, with the final annealing stage of $400-600{ }^{\circ} \mathrm{C}$ for the electrical conductivity of electron irradiated $n$-type GaAs [9], it should be noted that the recovery of electrical conductivity would largely be related to the annealing of point defects (interstitial vacancy recombination) and dopants while the nucleation and growth of small interstitial dislocation loops would mostly involve the diffusion and coalescence of $\mathrm{Ga}$ and $\mathrm{As}$ interstitials to sinks acting as nucleation centers for dislocation loops. The results of this paper indicate that this latter process (as observed by TEM) is what other workers [9] referred to as "disordered-region annealing" (from electrical conductivity measurements).

A HAADF STEM image of a small loop on a $\{111\}$ plane in neutron irradiated $n$-type GaAs is shown in Fig. 5a. A Burgers circuit drawn around the dislocation loop line reveals that the interstitial loop plane consists of one layer of $\mathrm{Ga}$ and As atoms while the stacking sequence across the loop is consistent with that of an extrinsic stacking fault [16]. The insertion of a single GaAs rotated layer results in the creation of two modified (rotated) layers, as can be seen in Fig. 5a and b at higher magnification. High resolution STEM-EELS elemental mapping was used to determine the polarity continuation across the dislocation loop. Figure 5c shows the positions of the $\mathrm{Ga}$ and As atoms across the extrinsic stacking fault plane of the $\{111\}$ loop obtained by using EEL spectrum imaging. In this configuration, polarity continuation across the loop is observed and charge neutrality is preserved. Future research will investigate whether the small $\{111\}$ loops were present in the $n$-type GaAs before irradiation and annealing.

\section{Summary and discussion}

This paper compares earlier TEM results of dislocation loops generated in $n$-type GaAs by $1 \mathrm{MeV}$ electron and fast neutron irradiation with recent Cs-corrected HAADF STEM imaging of $\{110\}$ and $\{111\}$ dislocation 
loops in neutron irradiated and annealed $n$-type GaAs. Cs-corrected HAADF STEM imaging is a powerful technique for determining the structural and chemical information of an extended defect at atomic resolution. In this paper Cs-corrected HAADF STEM was used to image the atomic structure of $\{110\}$ and $\{111\}$ dislocation loops in fast neutron irradiated $n$-type GaAs annealed at $600^{\circ} \mathrm{C}$ for $20 \mathrm{~min}$. To the best of our knowledge, this is the first TEM and HAADF STEM study of neutron irradiated GaAs to be published. A Burgers circuit drawn around the $\{110\}$ dislocation loop line indicates that the loop consists of two layers of GaAs atoms, which is consistent with the model for a $\{110\}$ pure-edge interstitial dislocation loop in GaAs.

A Burgers circuit drawn around the $\{111\}$ dislocation loop line revealed that the plane of the interstitial loop consists of one layer of $\mathrm{Ga}$ and As atoms while the stacking sequence across the loop is consistent with that of an extrinsic stacking fault. The insertion of a single GaAs rotated layer results in the creation of two modified (rotated) layers. High resolution STEM-EELS elemental mapping was used to determine the polarity continuation across the dislocation loop. The positions of the $\mathrm{Ga}$ and As atom columns across the extrinsic stacking fault plane of the $\{111\}$ loop indicate polarity continuation across the loop and the preservation of charge neutrality.

The annealing stage at $500^{\circ} \mathrm{C}$ found to be the minimum temperature for small dislocation loop formation visible in TEM, agrees with the final annealing stage of $400-600{ }^{\circ} \mathrm{C}$ for the electrical conductivity of electron irradiated $n$-type GaAs [9]. In the case of fast neutron irradiated $n$-type GaAs, a final annealing stage for electrical conductivity was found in the range $450-700^{\circ} \mathrm{C}[14]$.

Although the authors of the current paper associate the formation of dislocation loops observed by TEM at $500^{\circ} \mathrm{C}$ and above, with the final annealing stage of $400-600^{\circ} \mathrm{C}$ for the electrical conductivity of electron irradiated $n$-type GaAs, it should be noted that the recovery of electrical conductivity would largely be related to the annealing of point defects (interstitial-vacancy recombination) and dopants while the nucleation and growth of small interstitial dislocation loops would mostly involve the diffusion and coalescence of $\mathrm{Ga}$ and As interstitials to sinks acting as nucleation centres for dislocation loops.

Finally, with the recent data on dislocation loops in electron and neutron irradiated $n$-type GaAs, it is possible to reinterpret hydrogen platelets and $\{110\}$ interstitial loops as found in proton irradiated $n$ type GaAs. It is now proposed that $\{110\}$ loops in proton bombarded GaAs are the same type of interstitial loops found in neutron irradiated GaAs in this paper. Hydrogen platelets form on $\{111\}$ planes in GaAs exposed to a hydrogen plasma at $180^{\circ} \mathrm{C}$ [17] and in high-dose proton bombarded GaAs $\left(5 \times 10^{16}-10^{17}\right)$ annealed in the range $180-230^{\circ} \mathrm{C}$ [17]. Future research using HAADF STEM will investigate whether the small $\{111\}$ loops were present in the $n$-type GaAs before irradiation and annealing.

\section{Acknowledgments}

The National Research Foundation and Department of Science and Technology are acknowledged for financial support. The first author is grateful to Dr. F. Phillipp for the useful discussions and assistance with the use of the High Voltage Electron Microscope at the Max Planck Institute for Metals Research in Stuttgart some years ago.

\section{References}

[1] M. Yamaguchi, Solar Energy Mater. Solar Cells 68, 31 (2001).

[2] S. Moon, K. Kim, Y. Kim, J. Heo, J. Lee, Sci. Rep. 6, 30107 (2016).

[3] J.H. Neethling, H.C. Snyman, J. Appl. Phys. 60, 941 (1986).

[4] J.H. Neethling, H.C. Snyman, C.A.B. Ball, J. Appl. Phys. 63, 704 (1988).

[5] J.H. Neethling, Physica B 170, 285 (1991).

[6] G.H. Narayanan, A.H. Kachare, Phys. Status Solidi A 26, 657 (1974).

[7] D. Laister, G.M. Jenkins, Philos. Mag. 23, 1077 (1971).

[8] J. Borysiuk, J.A. Kozubowski, T. Slupinski, Acta Phys. Pol. A 90, 739 (1996).

[9] E.Y. Brailovskii, V.N. Broudnyi, A.A. Groza, Radiat. Dam. Def. Semicond. Inst. Phys. Conf. Ser. 16, $121(1973)$

[10] E.J. Olivier, J.H. Neethling, R.E. Kroon, S.R. Naidoo, C.S. Allen, H. Sawada, P.A. van Aken, A.I. Kirkland, Nature Mater. 17, 243 (2018).

[11] J.H. Neethling, in: Proc. 13th Int. Congress on Electron Microscopy, Paris, 1994, Vol. 2A, Eds. B. Jouffrey, C. Colliex, France, p. 101.

[12] H.C. Snyman, J.H Neethling, Physica 116B, 629 (1983).

[13] P.W. Hutchinson, P.S. Dobson, Philos. Mag. A 41, 601 (1980).

[14] L.W. Aukerman, P.W. Davis, R.D. Graft, T.S. Shilliday, J. Appl. Phys. 34, 3590 (1963).

[15] D.V. Lang, Radiat. Eff. Semicond. Inst. Phys. Conf. Ser.1, 70 (1977).

[16] J. Hornstra, J. Phys. Chem. Solids 5, 129 (1958).

[17] J.R. Botha, J.H. Neethling, A.W.R. Leitch, Semicond. Sci. Technol. 14, 1147 (1999). 